\title{
Existence and uniqueness of positive mild solutions for a class of fractional evolution equations on infinite interval
}

\author{
Yi Chen ${ }^{* *}$, Zhanmei Lv² and Liang Zhang ${ }^{3}$
}

${ }^{\text {*Correspondence: }}$
mathcyt@163.com
'School of Mathematics, China
University of Mining and
Technology, Xuzhou, 221116, China
Full list of author information is
available at the end of the article

available at the end of the article

\begin{abstract}
Based on an equivalent integral equation of a new type for a class of fractional evolution equations, which is different from those obtained in the existing literature, the paper investigates a class of fractional evolution equations with nonlocal conditions on infinite interval. Without the assumption of lower and upper solutions, we present a new result on the existence and uniqueness of positive mild solutions for the abstract fractional evolution equations by using the monotone iterative method.
\end{abstract}

Keywords: fractional order; abstract evolution equations; mild solutions; monotone iterative method

\section{Introduction}

Fractional calculus, a generalization of the ordinary differentiation and integration, has played a significant role in science, economy, engineering, biology, physics, and other fields (see [1-3]). Many real world phenomena and processes can be modeled as fractional differential equations, and due to the fact of their various applications in many fields, today, there is a large number of researchers turning to study the fractional differential equations. For more general theory of fractional differential equations, we refer readers to the papers [4-22] and the references given therein.

Among most of the studies on fractional differential equations, an important branch is devoted to investigating the fractional evolution equation, which is a valuable tool for describing physics phenomena. Recently, evolution equations of fractional order have attracted increasing attention, we refer to the papers [12-22] and the references therein.

In [13], EI-Borai investigated the Cauchy problem in a Banach space for fractional evolution equations. He has given an equivalent integral equation for a class of fractional evolution equations in terms of some probability densities by the method of Laplace transform. Based on the equivalent integral equation, EI-Borai has gained the existence and uniqueness results. Since then, most papers working on the evolution equations of fractional order, such as [14-22], have based their studies on this paper.

(c) The Author(s) 2017. This article is distributed under the terms of the Creative Commons Attribution 4.0 International License (http://creativecommons.org/licenses/by/4.0/), which permits unrestricted use, distribution, and reproduction in any medium, provided you give appropriate credit to the original author(s) and the source, provide a link to the Creative Commons license, and indicate if changes were made. 
In [23], Byszewski dealt with the following functional-differential abstract nonlocal Cauchy problem of integer order in a general Banach space:

$$
\left\{\begin{array}{l}
u^{\prime}(t)=f(t, u(t), u(a(t))), \quad t \in\left[t_{0}, t_{0}+T\right], \\
u\left(t_{0}\right)+\sum_{k=1}^{p} c_{k} u\left(t_{k}\right)=x_{0} .
\end{array}\right.
$$

Applying the Banach contraction theorem and a modified Picard method, the existence and uniqueness of a classical solution is given.

In [22], Zhou studied a class of Cauchy problems for fractional evolution equations with Caputo derivative on finite interval

$$
\left\{\begin{array}{l}
\left({ }^{C} D_{0+}^{\beta} x\right)(t)=A x(t)+(F x)(t), \quad t \in[0, a], \\
x(0)+g(x)=x_{0} .
\end{array}\right.
$$

According to the theory of Hausdorff measure of noncompactness and some fixed point theorems, Zhou has got the existence of the mild solution.

As far as we know, among the existing literature, a lot of works, such as [12-21], are focused on the existence of solutions for fractional evolution equations on finite interval. However, the existence results on the half-line are few.

Motivated by [13, 14, 22-24], in this paper, we are concerned with the following fractional evolution equation in a Banach space $E$ on the unbounded domains:

$$
\left\{\begin{array}{l}
{ }^{C} D_{0+}^{\beta} u(t)=A u(t)+\mathcal{F}(u)(t), \quad t \in(0,+\infty), \\
u(0)=\sum_{i=0}^{m} d_{i} u\left(\xi_{i}\right)
\end{array}\right.
$$

where ${ }^{C} D_{0+}^{\beta}$ is the Caputo fractional derivative, $0<\beta<1, d_{i}>0, \xi_{i}>0(i=0,1,2, \ldots), A$ is the infinitesimal generator of a $C_{0}$ semigroup $\{S(t)\}_{t \geq 0}$ of operators on Banach $E$, and $\mathcal{F}: \mathrm{BC}(J, E) \rightarrow \mathrm{BC}(J, E)$ is a given operator satisfying certain conditions, where $\mathrm{BC}(J, E)$ is a Banach space defined in the second part.

Utilizing similar methods to those used in [13,14], here we get a corrected type of the equivalent integral equation of (1.1), which is different from those gained in the existing literature. Without the assumption of lower and upper solutions, we present some new results on the existence of positive mild solutions for the abstract fractional evolution equations (1.1) by means of the monotone iterative method. And to our best knowledge, there is not any paper to deal with the abstract problems of fractional order on unbounded domains.

The rest of the paper is organized as follows. In Section 2, we introduce the definitions of semigroups, fractional integral and fractional derivative, some lemmas about fractional differential equations and some other preliminaries. In Section 3, we present the existence theorem of the solution for problem (1.1) by the monotone iterative method. Then an example is given in Section 4 to demonstrate the application of our result.

\section{Preliminaries}

First, let us recall some definitions and standard facts about the cone.

Let $P$ be a cone in the ordered Banach space $E$, which defines a partial order on $E$ by $x \leq y$ if and only if $y-x \in P . P$ is normal if there exists a positive constant $N$ such that 
$\theta \leq x \leq y$ implies $\|x\| \leq N\|y\|$, where $\theta$ is the zero element of the Banach space $E$. The infimum of all $N$ with the property above is called the normal constant of $P$. For more details of the cone $P$, we refer readers to $[25,26]$.

Throughout the paper, we set $E$ to be an ordered Banach space with the norm $\|\cdot\|$ and the partial order ' $\leq$ '. Let $P=\{x \in E \mid x \geq \theta\}$ be a positive cone, which is normal with normal constant $N$. Let $J=[0,+\infty)$. Set

$$
\mathrm{BC}(J, E)=\{u(t) \mid u(t) \text { is continuous and bounded on } J\} .
$$

Obviously, $\mathrm{BC}(J, E)$ is a Banach space with the norm $\|u\|^{*}=\sup _{t \in J}\|u(t)\|$. Let

$$
P^{*}=\{u \in \mathrm{BC}(J, E) \mid u(t) \geq \theta, t \in J\} .
$$

It is easy to see that $P^{*}$ is also normal with the same normal constant $N$ of the cone $P$. Besides, $\mathrm{BC}(J, E)$ is also an ordered Banach space with the partial order ' $\leq$ ' induced by the positive cone $P^{*}$ (without confusion, we denote by ' $\leq$ ' the partial order on both $E$ and $\mathrm{BC}(J, E))$.

We denote by $[v, w]$ the order interval $\left\{u \in P^{*} \mid v \leq u \leq w, v, w \in \mathrm{BC}(J, E)\right\}$ on $\mathrm{BC}(J, E)$ and use $[v(t), w(t)]$ to denote the order interval $\{z \in E \mid v(t) \leq z \leq w(t)\}$ on $E$ for $t \in J$.

Then we present some fundamental facts on the fractional calculus theory which we will use in the next section.

Definition 2.1 ([1-3]) The Riemann-Liouville fractional integral of order $v>0$ of a function $h:(0, \infty) \rightarrow \mathbb{R}$ is given by

$$
I_{0+}^{v} h(t)=D_{0+}^{-v} h(t)=\frac{1}{\Gamma(v)} \int_{0}^{t}(t-s)^{\nu-1} h(s) d s,
$$

provided that the right-hand side is pointwise defined on $(0, \infty)$.

Definition 2.2 ([1-3]) The Caputo fractional derivative of order $v>0$ of a continuous function $h:(0, \infty) \rightarrow \mathbb{R}$ is given by

$$
{ }^{C} D_{0+}^{v} h(t)=\frac{1}{\Gamma(n-v)} \int_{0}^{t}(t-s)^{n-v-1} h^{n}(s) d s
$$

where $n=[v]+1$, provided that the right-hand side is pointwise defined on $(0, \infty)$.

Lemma $2.1([1,3])$ Assume that ${ }^{C} D_{0+}^{v} h(t) \in L^{1}(0,+\infty), v>0$. Then we have

$$
I_{0+}^{v}{ }^{C} D_{0+}^{v} h(t)=h(t)+C_{1}+C_{2} t+\cdots+C_{N} t^{N-1}, \quad t>0,
$$

for some $C_{i} \in \mathbb{R}, i=1,2, \ldots, N$, where $N$ is the smallest integer greater than or equal to $v$.

If $h$ is an abstract function with values in a Banach space $E$, then the integrals appearing in Definition 2.1, Definition 2.2 and Lemma 2.1 are taken in Bochner's sense. And a measurable function $h$ is Bochner integrable if the norm of $h$ is Lebesgue integrable. 
Next, we give some facts about the semigroups of linear operators. These results can be found in $[27,28]$.

For a strongly continuous semigroup (i.e. $C_{0}$-semigroup) $\{S(t)\}_{t \geq 0}$, the infinitesimal generator of $\{S(t)\}_{t \geq 0}$ is defined by

$$
A x=\lim _{t \rightarrow 0^{+}} \frac{S(t) x-x}{t}, \quad x \in E .
$$

We denote by $D(A)$ the domain of $A$, that is,

$$
D(A)=\left\{x \in E \mid \lim _{t \rightarrow 0^{+}} \frac{S(t) x-x}{t} \text { exists }\right\}
$$

Lemma $2.2([27,28])$ Let $\{S(t)\}_{t \geq 0}$ be a $C_{0}$-semigroup, then there exist constants $C \geq 1$ and $\omega \in \mathbb{R}$ such that $\|S(t)\| \leq C e^{\omega t}, t \geq 0$.

Lemma $2.3([27,28])$ A linear operator $A$ is the infinitesimal generator of a $C_{0}$-semigroup $\{S(t)\}_{t \geq 0}$ if and only if

(i) $A$ is closed and $\overline{D(A)}=E$.

(ii) The resolvent set $\rho(A)$ of $A$ contains $\mathbb{R}^{+}$, and for every $\lambda>0$ we have

$$
\|R(\lambda, A)\| \leq \frac{1}{\lambda}
$$

where

$$
R(\lambda, A):=(\lambda I-A)^{-1}=\int_{0}^{+\infty} e^{-\lambda t} S(t) x d t, \quad x \in E .
$$

Definition 2.3 ([27, 28]) A $C_{0}$-semigroup $\{S(t)\}_{t \geq 0}$ is said to be uniformly exponentially stable if $\omega_{0}<0$, where $\omega_{0}$ is the growth bound of $\{S(t)\}_{t \geq 0}$, which is defined by

$$
\omega_{0}=\inf \left\{\omega \in \mathbb{R} \mid \exists C \geq 1 \text { such that }\|S(t)\| \leq C e^{\omega t}, t \geq 0\right\} .
$$

Definition 2.4 ([24]) A $C_{0}$-semigroup $\{S(t)\}_{t \geq 0}$ is said to be positive on $E$ if order inequality $S(t) x \geq \theta, x \in E$ and $t \geq 0$.

According to Lemma 2.2 and Definition 2.3, if $\{S(t)\}_{t \geq 0}$ is a uniformly exponentially stable $C_{0}$-semigroup with the growth bound $\omega_{0}$, then for any $\omega \in\left(0,\left|\omega_{0}\right|\right]$, there exists a constant $C \geq 1$ such that $\|S(t)\| \leq C e^{\omega t}, t \geq 0$. Now, we define a norm in $E$ by

$$
\|x\|_{\omega}=\sup _{t \geq 0}\left\|e^{\omega t} S(t) x\right\|
$$

Evidently, we have $\|x\| \leq\|x\|_{\omega} \leq C\|x\|$, that is to say, the norms $\|\cdot\|_{\omega}$ and $\|\cdot\|$ are equivalent. We denote by $\|S(t)\|_{\omega}$ the norm of $S(t)$ induced by the norm $\|\cdot\|_{\omega}$, then we obtain

$$
\|S(t)\|_{\omega} \leq e^{-\omega t}, \quad t \geq 0 .
$$


Also, we can define the equivalent norm on $\mathrm{BC}(J, E)$ by

$$
\|u\|_{\omega}^{*}=\sup _{t \in J}\|u(t)\|_{\omega}, \quad u \in \mathrm{BC}(J, E) .
$$

Consider the one-sided stable probability density $[14,15,29]$

$$
\zeta_{\beta}(\theta)=\frac{1}{\pi} \sum_{n=1}^{\infty} \theta^{-\beta n-1} \frac{\Gamma(n \beta+1)}{n !} \sin (n \pi \beta), \quad \theta \in(0, \infty)
$$

where $0<\beta<1$.

From $[14,15,29]$, the Laplace transform of the one-sided stable probability density $\zeta_{\beta}(\theta)$ is given by

$$
\mathcal{L}\left[\zeta_{\beta}(\theta)\right]=\int_{0}^{\infty} e^{-\lambda \theta} \zeta_{\beta}(\theta) d \theta=e^{-\lambda^{\beta}}, \quad 0<\beta<1
$$

By Remark 2.8 in [15], for $0 \leq \gamma \leq 1$, one has

$$
\int_{0}^{\infty} \theta^{-\beta \gamma} \zeta_{\beta}(\theta) d \theta=\frac{\Gamma(1+\gamma)}{\Gamma(1+\beta \gamma)}
$$

In the following, we assume that $\{S(t)\}_{t \geq 0}$ is a uniformly exponentially stable $C_{0}$ semigroup with the growth bound $\omega_{0}$, and $\omega \in\left(0,\left|\omega_{0}\right|\right]$.

Lemma 2.4 Let $\Psi: E \rightarrow E$

$$
\Psi x=\frac{\beta}{\Gamma(1-\beta)} \sum_{i=0}^{m} d_{i} \int_{0}^{1} \int_{0}^{\infty} \tau^{-\beta}(1-\tau)^{\beta-1} \frac{\zeta_{\beta}(\theta)}{\theta^{\beta}} S\left(\xi_{i}^{\beta} \frac{(1-\tau)^{\beta}}{\theta^{\beta}}\right) x d \theta d \tau .
$$

Then $\Psi$ is a linear bounded operator and $\|\Psi\|_{\omega} \leq \sum_{i=0}^{m} d_{i}$. Furthermore, if $0<\sum_{i=0}^{m} d_{i}<1$, then $(I-\Psi)^{-1}$ is also a linear bounded operator and

$$
\left\|(I-\Psi)^{-1}\right\|_{\omega} \leq \frac{1}{1-\sum_{i=0}^{m} d_{i}}
$$

Proof By the definition of $\Psi$, we have

$$
\begin{aligned}
\|\Psi x\|_{\omega} & \leq \frac{\beta}{\Gamma(1-\beta)} \sum_{i=0}^{m} d_{i} \int_{0}^{1} \int_{0}^{\infty} \tau^{-\beta}(1-\tau)^{\beta-1} \frac{\zeta_{\beta}(\theta)}{\theta^{\beta}}\left\|S\left(\frac{\xi_{i}^{\beta}(1-\tau)^{\beta}}{\theta^{\beta}}\right)\right\|_{\omega}\|x\|_{\omega} d \theta d \tau \\
& \leq \frac{\beta}{\Gamma(1-\beta)} \sum_{i=0}^{m}\left[d_{i} \int_{0}^{1} \int_{0}^{\infty} \tau^{-\beta}(1-\tau)^{\beta-1} \frac{\zeta_{\beta}(\theta)}{\theta^{\beta}} e^{\left.-\omega^{\frac{\xi_{i}^{\beta}(1-\tau)^{\beta}}{\theta^{\beta}}} d \theta d \tau\right]\|x\|_{\omega}}\right. \\
& \leq \frac{\beta}{\Gamma(1-\beta)}\left(\sum_{i=0}^{m} d_{i}\right)\left[\int_{0}^{1} \tau^{-\beta}(1-\tau)^{\beta-1}\left(\int_{0}^{\infty} \frac{\zeta_{\beta}(\theta)}{\theta^{\beta}} d \theta\right) d \tau\right]\|x\|_{\omega} \\
& \leq\left(\sum_{i=0}^{m} d_{i}\right)\|x\|_{\omega} .
\end{aligned}
$$

Thus, $\Psi$ is bounded and $\|\Psi\|_{\omega} \leq \sum_{i=0}^{m} d_{i}$. 
If $0<\sum_{i=0}^{m} d_{i}<1$, by the theory of linear operators, we can deduce that $(I-\Psi)^{-1}$ is also a linear bounded operator and

$$
\left\|(I-\Psi)^{-1}\right\|_{\omega} \leq \frac{1}{1-\sum_{i=0}^{m} d_{i}} .
$$

Lemma 2.5 Set

$$
(\Phi k)(t)=\beta \int_{0}^{t} \int_{0}^{\infty}(t-s)^{\beta-1} \frac{\zeta_{\beta}(\theta)}{\theta^{\beta}} S\left(\frac{(t-s)^{\beta}}{\theta^{\beta}}\right) k(s) d \theta d s, \quad k \in \mathrm{BC}(J, E) .
$$

Then $\Phi: \mathrm{BC}(J, E) \rightarrow \mathrm{BC}(J, E)$ and

$$
\|(\Phi k)\|_{\omega}^{*} \leq \frac{1}{\Gamma(\beta+1)} \frac{\|k\|_{\omega}^{*}}{\omega} .
$$

Proof Since

$$
\begin{aligned}
(\Phi k)(t) & =\beta \int_{0}^{t} \int_{0}^{\infty}(t-s)^{\beta-1} \frac{\zeta_{\beta}(\theta)}{\theta^{\beta}} S\left(\frac{(t-s)^{\beta}}{\theta^{\beta}}\right) k(s) d \theta d s \\
& =\beta \int_{0}^{1} \int_{0}^{\infty} t^{\beta}(1-\tau)^{\beta-1} \frac{\zeta_{\beta}(\theta)}{\theta^{\beta}} S\left(\frac{t^{\beta}(1-\tau)^{\beta}}{\theta^{\beta}}\right) k(t \tau) d \theta d \tau
\end{aligned}
$$

then

$$
\begin{aligned}
\|(\Phi k)(t)\|_{\omega} & \leq \beta \int_{0}^{1} \int_{0}^{\infty} t^{\beta}(1-\tau)^{\beta-1} \frac{\zeta_{\beta}(\theta)}{\theta^{\beta}}\left\|S\left(\frac{t^{\beta}(1-\tau)^{\beta}}{\theta^{\beta}}\right)\right\|_{\omega}\|k(t \tau)\|_{\omega} d \theta d \tau \\
& \leq \beta \int_{0}^{1} \int_{0}^{\infty} t^{\beta}(1-\tau)^{\beta-1} \frac{\zeta_{\beta}(\theta)}{\theta^{\beta}} e^{-\omega \frac{t^{\beta}(1-\tau)^{\beta}}{\theta^{\beta}}}\|k(t \tau)\|_{\omega} d \theta d \tau \\
& \leq \frac{\|k\|_{\omega}^{*}}{\omega} \int_{0}^{\infty}\left[\left(\int_{0}^{1} e^{\left.-\omega \frac{t^{\beta}(1-\tau)}{\theta^{\beta}}\right)} d\left(-\omega \frac{t^{\beta}(1-\tau)^{\beta}}{\theta^{\beta}}\right)\right) \frac{\zeta_{\beta}(\theta)}{\theta^{\beta}}\right] d \theta \\
& =\frac{\|k\|_{\omega}^{*}}{\omega} \int_{0}^{\infty}\left(1-e^{\left.-\omega \frac{t^{\beta}}{\theta^{\beta}}\right)} \frac{\zeta_{\beta}(\theta)}{\theta^{\beta}} d \theta\right. \\
& \leq \frac{1}{\Gamma(\beta+1)} \frac{\|k\|_{\omega}^{*}}{\omega} .
\end{aligned}
$$

Hence,

$$
\|(\Phi k)\|_{\omega}^{*} \leq \frac{1}{\Gamma(\beta+1)} \frac{\|k\|_{\omega}^{*}}{\omega} .
$$

\section{Main results}

In this section, we will present the existence theorem for the abstract fractional differential equation on the half-line. In order to prove our main result, we need the following facts and lemmas.

Lemma 3.1 Let $k \in \mathrm{BC}(J, E)$ and $0<\sum_{i=0}^{m} d_{i}<1$. Then, for given $u_{0} \in D(A)$, the following linear equation

$$
\left\{\begin{array}{l}
{ }^{C} D_{0+}^{\beta} u(t)=A u(t)+k(t), \quad t \in(0,+\infty) \\
u(0)=u_{0}
\end{array}\right.
$$


has a unique mild solution $u \in \mathrm{BC}(J, E)$ such that

$$
\begin{aligned}
u(t) & =\int_{0}^{t} \int_{0}^{\infty}\left[\beta \frac{(t-s)^{\beta-1}}{\theta^{\beta}} \zeta_{\beta}(\theta) S\left(\frac{(t-s)^{\beta}}{\theta^{\beta}}\right)\left(\frac{s^{-\beta}}{\Gamma(1-\beta)} u_{0}+k(s)\right)\right] d \theta d s \\
& =\frac{\beta}{\Gamma(1-\beta)} \int_{0}^{1} \int_{0}^{\infty} \frac{\zeta_{\beta}(\theta)}{\theta^{\beta}} \tau^{-\beta}(1-\tau)^{\beta-1} S\left(\frac{t^{\beta}(1-\tau)^{\beta}}{\theta^{\beta}}\right) u_{0} d \theta d \tau+(\Phi k)(t) .
\end{aligned}
$$

Proof Employing Lemma 2.1, one can rewrite (3.1) as the following equivalent integral equation:

$$
u(t)=u_{0}+\frac{1}{\Gamma(\beta)} \int_{0}^{t}(t-s)^{\beta-1}[A u(s)+k(s)] d s
$$

With an approach similar to $[12,13]$, by using the Laplace transform, we can get

$$
U(\lambda)=\lambda^{-1} u_{0}+\lambda^{-\beta} A U(\lambda)+\lambda^{-\beta} K(\lambda), \quad \lambda>0,
$$

where $U(\lambda)$ and $K(\lambda)$ is the Laplace transform of $u(t)$ and $k(t)$, respectively.

Then (3.4) leads to

$$
\left(\lambda^{\beta} I-A\right) U(\lambda)=\lambda^{\beta-1} u_{0}+K(\lambda)
$$

In view of (2.5) and Lemma 2.3, we have

$$
\begin{aligned}
U(\lambda)= & \left(\lambda^{\beta} I-A\right)^{-1} \lambda^{\beta-1} u_{0}+\left(\lambda^{\beta} I-A\right)^{-1} K(\lambda) \\
= & \lambda^{\beta-1} \int_{0}^{\infty} e^{-\lambda^{\beta} s} S(s) u_{0} d s+\int_{0}^{\infty} e^{-\lambda^{\beta} s} S(s) K(\lambda) d s \\
= & \lambda^{\beta-1} \int_{0}^{\infty} \int_{0}^{\infty} e^{-\lambda s^{1 / \beta} \theta} \zeta_{\beta}(\theta) S(s) u_{0} d \theta d s \\
& +\int_{0}^{\infty} \int_{0}^{\infty} e^{-\lambda s^{1 / \beta} \theta} \zeta_{\beta}(\theta) S(s) K(\lambda) d \theta d s \\
= & \lambda^{\beta-1} \int_{0}^{\infty} e^{-\lambda t}\left[\int_{0}^{\infty} \beta \frac{t^{\beta-1}}{\theta^{\beta}} \zeta_{\beta}(\theta) S\left(\frac{t^{\beta}}{\theta^{\beta}}\right) u_{0} d \theta\right] d t \\
& +\int_{0}^{\infty} e^{-\lambda t}\left[\int_{0}^{t} \int_{0}^{\infty} \beta \frac{(t-s)^{\beta-1}}{\theta^{\beta}} \zeta_{\beta}(\theta) S\left(\frac{(t-s)^{\beta}}{\theta^{\beta}}\right) k(s) d \theta d s\right] d t .
\end{aligned}
$$

With the help of the convolution theorem, using the inverse Laplace transforms and Lemma 2.5 , one can derive that

$$
\begin{aligned}
u(t)= & \mathcal{L}^{-1}\left[\lambda^{\beta-1}\right] *\left[\int_{0}^{\infty} \beta \frac{t^{\beta-1}}{\theta^{\beta}} \zeta_{\beta}(\theta) S\left(\frac{t^{\beta}}{\theta^{\beta}}\right) u_{0} d \theta\right] \\
& +\int_{0}^{t} \int_{0}^{\infty} \beta \frac{(t-s)^{\beta-1}}{\theta^{\beta}} \zeta_{\beta}(\theta) S\left(\frac{(t-s)^{\beta}}{\theta^{\beta}}\right) k(s) d \theta d s \\
= & \frac{t^{-\beta}}{\Gamma(1-\beta)} *\left[\int_{0}^{\infty} \beta \frac{t^{\beta-1}}{\theta^{\beta}} \zeta_{\beta}(\theta) S\left(\frac{t^{\beta}}{\theta^{\beta}}\right) u_{0} d \theta\right] \\
& +\beta \int_{0}^{t} \int_{0}^{\infty} \frac{(t-s)^{\beta-1}}{\theta^{\beta}} \zeta_{\beta}(\theta) S\left(\frac{(t-s)^{\beta}}{\theta^{\beta}}\right) k(s) d \theta d s
\end{aligned}
$$




$$
\begin{aligned}
& =\frac{\beta}{\Gamma(1-\beta)} \int_{0}^{t} \int_{0}^{\infty} s^{-\beta} \frac{(t-s)^{\beta-1}}{\theta^{\beta}} \zeta_{\beta}(\theta) S\left(\frac{(t-s)^{\beta}}{\theta^{\beta}}\right) u_{0} d \theta d s+(\Phi k)(t) \\
& =\frac{\beta}{\Gamma(1-\beta)} \int_{0}^{1} \int_{0}^{\infty} \frac{\zeta_{\beta}(\theta)}{\theta^{\beta}} \tau^{-\beta}(1-\tau)^{\beta-1} S\left(\frac{t^{\beta}(1-\tau)^{\beta}}{\theta^{\beta}}\right) u_{0} d \theta d \tau+(\Phi k)(t) .
\end{aligned}
$$

Since

$$
\begin{aligned}
\frac{\beta}{\Gamma(1-\beta)} \int_{0}^{1} \int_{0}^{\infty} \tau^{-\beta}(1-\tau)^{\beta-1} \frac{\zeta_{\beta}(\theta)}{\theta^{\beta}}\left\|S\left(\frac{t^{\beta}(1-\tau)^{\beta}}{\theta^{\beta}}\right) u_{0}\right\|_{\omega} d \theta d \tau \\
\quad+\|(\Phi k)(t)\|_{\omega} \\
\leq \frac{\beta}{\Gamma(1-\beta)} \int_{0}^{1} \int_{0}^{\infty} \tau^{-\beta}(1-\tau)^{\beta-1} \frac{\zeta_{\beta}(\theta)}{\theta^{\beta}} e^{\left.-\omega \frac{t^{\beta}(1-\tau)^{\beta}}{\theta^{\beta}}\right)}\left\|u_{0}\right\|_{\omega} d \theta d \tau \\
\quad+\|(\Phi k)(t)\|_{\omega} \\
\leq \frac{\beta}{\Gamma(1-\beta)}\left\|u_{0}\right\|_{\omega} \int_{0}^{1} \tau^{-\beta}(1-\tau)^{\beta-1}\left(\int_{0}^{\infty} \frac{\zeta_{\beta}(\theta)}{\theta^{\beta}} d \theta\right) d \tau+\|(\Phi k)(t)\|_{\omega} \\
\leq\left\|u_{0}\right\|_{\omega}+\frac{1}{\Gamma(\beta+1)} \frac{\|k\|_{\omega}^{*}}{\omega} .
\end{aligned}
$$

So, $u \in \mathrm{BC}(J, E)$.

Define an operator $\mathcal{B}_{A}$ on $\mathrm{BC}(J, E)$ as

$$
\begin{aligned}
\left(\mathcal{B}_{A} k\right)(t)= & \int_{0}^{t} \int_{0}^{\infty}\left[\beta \frac{(t-s)^{\beta-1}}{\theta^{\beta}} \zeta_{\beta}(\theta) S\left(\frac{(t-s)^{\beta}}{\theta^{\beta}}\right)\right. \\
& \left.\times\left(\frac{s^{-\beta}}{\Gamma(1-\beta)}\left[(I-\Psi)^{-1} \sum_{i=0}^{m} d_{i}(\Phi k)\left(\xi_{i}\right)\right]+k(s)\right)\right] d \theta d s
\end{aligned}
$$

where $k \in \mathrm{BC}(J, E)$.

For simplicity of notation, we denote

$$
\sigma:=\frac{1}{\Gamma(\beta+1)}\left(\frac{1}{1-\sum_{i=0}^{m} d_{i}}\right) .
$$

Lemma 3.2 Let $k \in \mathrm{BC}(J, E)$ and $0<\sum_{i=0}^{m} d_{i}<1$. Then, for given $u_{0} \in D(A)$, the following linear equation

$$
\left\{\begin{array}{l}
{ }^{C} D_{0+}^{\beta} u(t)=A u(t)+k(t), \quad t \in(0,+\infty), \\
u(0)=\sum_{i=0}^{m} d_{i} u\left(\xi_{i}\right)
\end{array}\right.
$$

has a unique mild solution $u \in \mathrm{BC}(J, E)$ such that

$$
\begin{aligned}
u(t)= & \left(\mathcal{B}_{A} k\right)(t) \\
= & \int_{0}^{1} \int_{0}^{\infty} \frac{\beta}{\Gamma(1-\beta)} \frac{\zeta_{\beta}(\theta)}{\theta^{\beta}} \tau^{-\beta}(1-\tau)^{\beta-1} S\left(t^{\beta} \frac{(1-\tau)^{\beta}}{\theta^{\beta}}\right) \\
& \times\left[(I-\Psi)^{-1} \sum_{i=0}^{m} d_{i}(\Phi k)\left(\xi_{i}\right)\right] d \theta d \tau+(\Phi k)(t) .
\end{aligned}
$$


Moreover,

$$
\left\|\mathcal{B}_{A}\right\|_{\omega}^{*} \leq \frac{\sigma}{\omega} .
$$

Proof From Lemma 3.1, we can have

$$
u\left(\xi_{i}\right)=\int_{0}^{\xi_{i}} \int_{0}^{\infty}\left[\beta \frac{\left(\xi_{i}-s\right)^{\beta-1}}{\theta^{\beta}} \zeta_{\beta}(\theta) S\left(\frac{\left(\xi_{i}-s\right)^{\beta}}{\theta^{\beta}}\right)\left(\frac{s^{-\beta}}{\Gamma(1-\beta)} u(0)+k(s)\right)\right] d \theta d s .
$$

By the nonlocal condition of (3.5), we get

$$
\begin{aligned}
u(0)= & \frac{\beta}{\Gamma(1-\beta)} \sum_{i=0}^{m} d_{i} \int_{0}^{\xi_{i}} \int_{0}^{\infty} \frac{\zeta_{\beta}(\theta)}{\theta^{\beta}} s^{-\beta}\left(\xi_{i}-s\right)^{\beta-1} S\left(\frac{\left(\xi_{i}-s\right)^{\beta}}{\theta^{\beta}}\right) u(0) d \theta d s \\
& +\sum_{i=0}^{m} d_{i}(\Phi k)\left(\xi_{i}\right) \\
= & \frac{\beta}{\Gamma(1-\beta)} \sum_{i=0}^{m} d_{i} \int_{0}^{1} \int_{0}^{\infty} \frac{\zeta_{\beta}(\theta)}{\theta^{\beta}} \tau^{-\beta}(1-\tau)^{\beta-1} S\left(\xi_{i}^{\beta} \frac{(1-\tau)^{\beta}}{\theta^{\beta}}\right) u(0) d \theta d \tau \\
& +\sum_{i=0}^{m} d_{i}(\Phi k)\left(\xi_{i}\right) \\
= & \Psi u(0)+\sum_{i=0}^{m} d_{i}(\Phi k)\left(\xi_{i}\right) .
\end{aligned}
$$

Thus,

$$
(I-\Psi) u(0)=\sum_{i=0}^{m} d_{i}(\Phi k)\left(\xi_{i}\right) .
$$

So, one can obtain

$$
u(0)=(I-\Psi)^{-1} \sum_{i=0}^{m} d_{i}(\Phi k)\left(\xi_{i}\right)
$$

Then, combined with Lemma 3.1, we can get

$$
\begin{aligned}
u(t)= & \int_{0}^{1} \int_{0}^{\infty} \frac{\beta}{\Gamma(1-\beta)} \frac{\zeta_{\beta}(\theta)}{\theta^{\beta}} \tau^{-\beta}(1-\tau)^{\beta-1} S\left(t^{\beta} \frac{(1-\tau)^{\beta}}{\theta^{\beta}}\right) \\
& \times\left[(I-\Psi)^{-1} \sum_{i=0}^{m} d_{i}(\Phi k)\left(\xi_{i}\right)\right] d \theta d \tau+(\Phi k)(t) .
\end{aligned}
$$

By virtue of (3.6), one has

$$
\begin{aligned}
\left\|\left(\mathcal{B}_{A} k\right)(t)\right\|_{\omega} \leq & \int_{0}^{1} \int_{0}^{\infty} \frac{\beta}{\Gamma(1-\beta)} \frac{\zeta_{\beta}(\theta)}{\theta^{\beta}} \tau^{-\beta}(1-\tau)^{\beta-1}\left\|S\left(t^{\beta} \frac{(1-\tau)^{\beta}}{\theta^{\beta}}\right)\right\|_{\omega} \\
& \left\|(I-\Psi)^{-1} \sum_{i=0}^{m} d_{i}(\Phi k)\left(\xi_{i}\right)\right\|_{\omega} d \theta d \tau+\|(\Phi k)(t)\|_{\omega}
\end{aligned}
$$




$$
\begin{aligned}
\leq & \int_{0}^{1} \int_{0}^{\infty} \frac{\beta}{\Gamma(1-\beta)} \frac{\zeta_{\beta}(\theta)}{\theta^{\beta}} \tau^{-\beta}(1-\tau)^{\beta-1} e^{-\omega^{t^{\beta}(1-\tau)^{\beta}}} \\
& \|\left[(I-\Psi)^{-1}\left\|_{\omega}\right\|(I-\Psi)^{-1} \sum_{i=0}^{m} d_{i}(\Phi k)\left(\xi_{i}\right)\left\|_{\omega} d \theta d \tau+\right\|(\Phi k)(t) \|_{\omega}\right. \\
\leq & \frac{1}{1-\sum_{i=0}^{m} d_{i}} \frac{\sum_{i=0}^{m} d_{i}}{\Gamma(\beta+1)} \frac{\|k\|_{\omega}^{*}}{\omega}+\frac{1}{\Gamma(\beta+1)} \frac{\|k\|_{\omega}^{*}}{\omega} \\
= & \frac{1}{\Gamma(\beta+1)} \frac{1}{1-\sum_{i=0}^{m} d_{i}} \frac{\|k\|_{\omega}^{*}}{\omega} \\
= & \frac{\sigma}{\omega}\|k\|_{\omega}^{*} .
\end{aligned}
$$

Then

$$
\left\|\left(\mathcal{B}_{A} k\right)\right\|_{\omega}^{*} \leq \frac{\sigma}{\omega}\|k\|_{\omega}^{*} .
$$

Next, we list some conditions that will be used in our main theorem as follows:

(H1) There exists a constant $\mathcal{P}<-\omega_{0}$ such that for

$$
\mathcal{F}(v)-\mathcal{F}(u) \leq \mathcal{P}(v-u), \quad \theta \leq u \leq v
$$

(H2) There exists a constant $\mathcal{Q}>\max \left\{-\mathcal{P}, \omega_{0}\right\}$ such that for

$$
\mathcal{F}(v)-\mathcal{F}(u) \geq-\mathcal{Q}(v-u), \quad \theta \leq u \leq v .
$$

$$
0<\frac{\mathcal{P}+\mathcal{Q}}{\mathcal{Q}-\omega_{0}}<\frac{1}{\sigma}
$$

Now, it is time for us to state the main result about the existence of positive solutions to problem (1.1) in the following.

Theorem 3.1 Let $\{S(t)\}_{t \geq 0}$ be a uniformly exponentially stable $C_{0}$-semigroup on a Banach space $E$ with the growth bound $\omega_{0}\left(\omega_{0}<0\right)$, and $A$ is the infinitesimal generator of $\{S(t)\}_{t \geq 0}$. Let $0<\sum_{i=0}^{m} d_{i}<1$. Assume that $P$ is a positive normal cone on $E$ with $N$ as its normal constant. Provided that $\mathcal{F}: \mathrm{BC}(J, E) \rightarrow \mathrm{BC}(J, E)$ is continuous and $f_{0}(t):=\mathcal{F}(\theta) \geq \theta$ is bounded on J. If $\mathcal{F}(u)$ satisfies conditions $(H 1),(H 2)$ and (H3), then problem (1.1) has a unique positive mild solution in $\mathrm{BC}(J, E)$.

Proof The proof is divided into three parts.

Part 1: Investigate two linear fractional evolution equations.

Consider two abstract fractional differential equations as follows:

$$
\left\{\begin{array}{l}
{ }^{C} D_{0+}^{\beta} u(t)=A u(t)+\mathcal{P} u(t)+f_{0}(t), \quad t \in(0,+\infty), \\
u(0)=\sum_{i=0}^{m} d_{i} u\left(\xi_{i}\right),
\end{array}\right.
$$


and

$$
\left\{\begin{array}{l}
{ }^{C} D_{0+}^{\beta} u(t)+\mathcal{Q} u(t)=A u(t)+h(t), \quad t \in(0,+\infty), \\
u(0)=\sum_{i=0}^{m} d_{i} u\left(\xi_{i}\right)
\end{array}\right.
$$

where $h \in \mathrm{BC}(J, E)$ is a given function.

By virtue of the theory of semigroups, it is obvious that $\left\{e^{\mathcal{P} t} S(t)\right\}_{t \geq 0}$ is a uniformly exponentially stable $C_{0}$-semigroup on Banach $E$ generated by $A+\mathcal{P} I$, and $A-\mathcal{Q} I$ generates a uniformly exponentially stable $C_{0}$-semigroup $\left\{e^{\mathcal{Q} t} S(t)\right\}_{t \geq 0}$ on Banach $E$. Both of the semigroups are positive, with the growth bounds $\mathcal{P}+\omega_{0}\left(\mathcal{P}+\omega_{0}<0\right)$ and $-\mathcal{Q}+\omega_{0}\left(-\mathcal{Q}+\omega_{0}<0\right)$, respectively.

In view of Lemma 3.2, equation (3.7) has a unique mild solution $\eta_{0} \in \mathrm{BC}(J, E)$. Moreover, $\eta_{0} \geq \theta$ due to the fact that $f_{0}(t) \geq \theta, t \in J$.

In consideration of Lemma 3.2, the unique mild solution of (3.8) can be written as $u=$ $\mathcal{B}_{A-\mathcal{Q} I} h$, where $\mathcal{B}_{A-\mathcal{Q} I}: \mathrm{BC}(J, E) \rightarrow \mathrm{BC}(J, E)$, analogous to the linear bounded operator $L_{A}$, is a positive bounded linear operator with the property that

$$
\left\|\mathcal{B}_{A-\mathcal{Q} I}\right\|_{\omega}^{*} \leq \frac{\sigma}{\mathcal{Q}-\omega_{0}}, \quad \omega=\mathcal{Q}-\omega_{0}
$$

In light of the above facts, one can deduce that $\eta_{0}$ is the mild solution of problem (3.8) for $h=f_{0}+\mathcal{P} \eta_{0}+\mathcal{Q} \eta_{0}$, so

$$
\eta_{0}=\mathcal{B}_{A-\mathcal{Q} I}\left(f_{0}+\mathcal{P} \eta_{0}+\mathcal{Q} \eta_{0}\right)
$$

Part 2: Prove the existence of mild solutions for problem (1.1).

Let $G(u)=\mathcal{F}(u)+\mathcal{Q} u$. It is clear that $G(\theta)=\mathcal{F}(\theta)=f_{0} \geq \theta$ and $G: \mathrm{BC}(J, E) \rightarrow \mathrm{BC}(J, E)$ is continuous as a result of the continuity of $\mathcal{F}$ and conditions $(H 1)$ and $(H 2)$.

In view of $(H 2)$, one can get that

$$
G(v)-G(u)=\mathcal{F}(v)+\mathcal{Q} v-\mathcal{F}(u)-\mathcal{Q} u=\mathcal{F}(v)-\mathcal{F}(u)+\mathcal{Q}(v-u) \geq \theta, \quad \theta \leq u \leq v,
$$

which shows that $G$ is an increasing operator on the positive cone $P^{*}$.

Let $\mathcal{M}=\mathcal{B}_{A-\mathcal{Q} I} \circ \mathrm{G}$. It is easy to see that the fixed point of the composition operator $\mathcal{M}$ is the mild solution of problem (1.1). Below we are going to prove that the operator $\mathcal{M}$ has at least one fixed point by the monotone iterative method.

Now, we define two sequences

$$
\eta_{n}=\mathcal{M}\left(\eta_{n-1}\right), \quad n=1,2,3, \ldots
$$

and

$$
\varpi_{0}=\theta, \quad \varpi_{n}=\mathcal{M}\left(\varpi_{n-1}\right), \quad n=1,2,3, \ldots
$$

By $(H 1)$, we have

$$
\mathcal{F}\left(\eta_{0}\right)-\mathcal{F}(\theta) \leq \mathcal{P} \eta_{0},
$$


that is,

$$
\mathcal{F}\left(\eta_{0}\right) \leq \mathcal{P} \eta_{0}+f_{0}
$$

Thus, we obtain

$$
\theta \leq G(\theta) \leq G\left(\eta_{0}\right)=\mathcal{F}\left(\eta_{0}\right)+\mathcal{Q} \eta_{0} \leq \mathcal{P} \eta_{0}+\mathcal{Q} \eta_{0}+f_{0} .
$$

Combined with (3.9) and the positivity of $\mathcal{B}_{A-\mathcal{Q} I}$, we get

$$
\theta \leq\left(\mathcal{B}_{A-\mathcal{Q} I} \circ G\right)(\theta)=\mathcal{M}(\theta) \leq\left(\mathcal{B}_{A-\mathcal{Q} I} \circ G\right)\left(\eta_{0}\right)=\mathcal{M}\left(\eta_{0}\right) \leq \mathcal{B}_{A-\mathcal{Q} I}\left(\mathcal{P} \eta_{0}+\mathcal{Q} \eta_{0}+f_{0}\right)=\eta_{0}
$$

that is,

$$
\theta=\varpi_{0} \leq \eta_{1} \leq \eta_{0}
$$

Since $\mathcal{M}$ is an increasing operator on the order interval $\left[\theta, \eta_{0}\right]$, by the definition of $\mathcal{M}$ and (3.12), we can obtain two sequences $\left\{\eta_{n}\right\}$ and $\left\{\varpi_{n}\right\}(n=0,1,2,3, \ldots)$ satisfying

$$
\theta=\varpi_{0} \leq \varpi_{1} \leq \varpi_{2} \leq \cdots \leq \varpi_{n} \leq \cdots \leq \eta_{n} \leq \cdots \leq \eta_{2} \leq \eta_{1} \leq \eta_{0} .
$$

Then, from $(H 1)$, one can get that

$$
\begin{aligned}
\theta & \leq \eta_{n}-\varpi_{n}=\mathcal{M}\left(\eta_{n-1}\right)-\mathcal{M}\left(\varpi_{n-1}\right)=\left(\mathcal{B}_{A-\mathcal{Q} I} \circ G\right)\left(\eta_{n-1}\right)-\left(\mathcal{B}_{A-\mathcal{Q} I} \circ G\right)\left(\varpi_{n-1}\right) \\
& =\mathcal{B}_{A-\mathcal{Q} I}\left[f\left(\cdot, \eta_{n-1}\right)+\mathcal{Q} \eta_{n-1}-f\left(\cdot, \varpi_{n-1}\right)-\mathcal{Q} \varpi_{n-1}\right] \\
& \leq(\mathcal{P}+\mathcal{Q}) \mathcal{B}_{A-\mathcal{Q} I}\left(\eta_{n-1}-\varpi_{n-1}\right) .
\end{aligned}
$$

Then, through mathematical induction, we have

$$
\theta \leq \eta_{n}-\varpi_{n} \leq(\mathcal{P}+\mathcal{Q})^{n} \mathcal{B}_{A-\mathcal{Q} I}^{n}\left(\eta_{0}-\varpi_{0}\right)=(\mathcal{P}+\mathcal{Q})^{n} \mathcal{B}_{A-\mathcal{Q} I}^{n}\left(\eta_{0}\right)
$$

As the cone $P^{*}$ is normal with the normal constant $N$, in view of $(H 3)$, we can get

$$
\begin{aligned}
\left\|\eta_{n}-\varpi_{n}\right\|_{\omega}^{*} & \leq N(\mathcal{P}+\mathcal{Q})^{n}\left\|\mathcal{B}_{A-\mathcal{Q} I}^{n}\left(\eta_{0}\right)\right\|_{\omega}^{*} \leq N(\mathcal{P}+\mathcal{Q})^{n}\left\|\mathcal{B}_{A-\mathcal{Q} I}^{n}\right\|_{\omega}^{*}\left\|\eta_{0}\right\|_{\omega}^{*} \\
& \leq N(\mathcal{P}+\mathcal{Q})^{n}\left(\left\|\mathcal{B}_{A-\mathcal{Q} I}\right\|^{*}\right)^{n}\left\|\eta_{0}\right\|_{\omega}^{*} \\
& \leq N\left(\mathcal{P}+\mathcal{K}_{2}\right)^{n}\left(\frac{\sigma}{\mathcal{Q}-\omega_{0}}\right)^{n}\left\|\eta_{0}\right\|_{\omega}^{*} \\
& =N\left(\frac{\sigma(\mathcal{P}+\mathcal{Q})}{\mathcal{Q}-\omega_{0}}\right)^{n}\left\|\eta_{0}\right\|_{\omega}^{*} \rightarrow 0, \quad n \rightarrow+\infty
\end{aligned}
$$

Analogous to the nested interval method, by virtue of (3.13), we can prove that there exists a unique mild solution $u^{*} \in \bigcap_{n=1}^{\infty}\left[\varpi_{n}, \eta_{n}\right]$ such that

$$
u^{*}=\lim _{n \rightarrow \infty} \eta_{n}=\lim _{n \rightarrow \infty} \varpi_{n} .
$$


By taking limit of $n$ tending to $+\infty$ on both of (3.10) and (3.11), one can obtain

$$
u^{*}=\mathcal{M}\left(u^{*}\right)
$$

which shows that $u^{*}$ is the fixed point of $\mathcal{M}$. Therefore, $u^{*}$ is a mild positive solution of problem (1.1).

Part 3: Certify the uniqueness of the mild solution for problem (1.1).

In this part, we will discuss the uniqueness of the mild solution for problem (1.1) by reduction to absurdity.

Suppose that $u_{1}^{*}$ and $u_{2}^{*}$ are two different positive mild solution for the fractional evolution equation (1.1), that is, $\left\|u_{1}^{*}-u_{2}^{*}\right\|_{\omega}^{*}>0$.

By the same steps as above, replacing $\eta_{0}$ by $u_{1}^{*}$ and $u_{2}^{*}$ in (3.10), respectively, then one can get that

$$
u_{i}^{*}=\mathcal{M}\left(u_{i}^{*}\right), \quad\left\|u_{i}^{*}-\varpi_{n}\right\|^{*} \rightarrow 0 \quad(n \rightarrow \infty), \quad \eta_{n}=u_{i}^{*}, \quad n \in \mathbb{N}, i=1,2 .
$$

Hence,

$$
0<\left\|u_{1}^{*}-u_{2}^{*}\right\|_{\omega}^{*} \leq\left\|u_{1}^{*}-\varpi_{n}\right\|_{\omega}^{*}+\left\|u_{2}^{*}-\varpi_{n}\right\|_{\omega}^{*} \rightarrow 0, \quad n \rightarrow \infty,
$$

which is a contradiction.

Therefore, problem (1.1) has a unique positive solution. The proof is completed.

\section{Examples}

To illustrate our main result, we will present an example.

Consider the following partial fractional differential equation:

\section{Example 4.1}

$$
\left\{\begin{array}{l}
\partial_{t}^{\beta} y(t, x)=\partial_{x}^{2} y(t, x)+L(t, y(t, x)), \quad t \in[0,+\infty) \\
y(t, 0)=y(t, \pi)=0, \quad t \in[0,+\infty), \\
y(0, x)=\sum_{i=0}^{m} d_{i} y\left(\xi_{i}, x\right), \quad x \in[0, \pi]
\end{array}\right.
$$

where $\partial_{t}^{\beta}$ is the Caputo fractional partial derivative of order $\beta \in(0,1)$.

Set $E=L^{2}([0, \pi], \mathbb{R})$ and $A y=\partial_{x}^{2} y$, then $A: D(A) \rightarrow E$ is a linear operator with domain $D(A)=\left\{u \in E \mid u^{\prime} \in E, u(0)=u(\pi)=0\right\}$. According to [30], the operator $A$ generates a uniformly exponentially stable $C_{0}$-semigroup $\{S(t)\}_{t \geq 0}$ with the growth bound $\omega_{0} \leq-1$.

Let $u(t)=y(t, \cdot), \mathcal{F}(u)(t)=L(t, y(t, \cdot))$, then we can rewrite problem (4.1) as

$$
\left\{\begin{array}{l}
{ }^{C} D_{0+}^{\beta} u(t)=A u(t)+\mathcal{F}(u)(t), \quad t \in(0,+\infty), \\
u(0)=\sum_{i=0}^{m} d_{i} u\left(\xi_{i}\right)
\end{array}\right.
$$

Take $\beta=1 / 2, m=6, d_{i}>0(i=0,1, \ldots, 6), \sum_{i=0}^{6} d_{i}=2 / 3, \xi_{i}>1$, then we can get

$$
\sigma=\frac{1}{\Gamma(\beta+1)}\left(\frac{1}{1-\sum_{i=0}^{m} d_{i}}\right)=\frac{6}{\sqrt{\pi}}
$$


Set

$$
\mathcal{F}(u)(t)=\left(-\mathcal{Q}+\frac{1}{p^{2}(t)}\right) u,
$$

where $p \in C[0,+\infty)$ is bounded.

Let

$$
\mathcal{P}=2 \omega_{0}, \quad \mathcal{Q}=-\left(2+\frac{2}{\sigma-1}\right) \omega_{0},
$$

which shows that

$$
0<\frac{\mathcal{P}+\mathcal{Q}}{\mathcal{Q}-\omega_{0}}=\frac{2}{3 \sigma-1}<\frac{1}{\sigma} .
$$

For

$$
-\mathcal{Q}-\frac{\omega_{0}}{\sigma-1}=\left(2+\frac{2}{\sigma-1}\right) \omega_{0}-\frac{\omega_{0}}{\sigma-1} \leq 2 \omega_{0}=\mathcal{P}
$$

then

$$
\begin{aligned}
-\mathcal{Q} & \leq \mathcal{F}(v)-\mathcal{F}(u)=\left[-\mathcal{Q}+\frac{-\omega_{0}}{\left(1+p^{2}(t)\right)(\sigma-1)}\right](v-u) \\
& \leq\left(-\mathcal{Q}-\frac{\omega_{0}}{\sigma-1}\right)(v-u) \\
& \leq \mathcal{P}(v-u), \quad \theta \leq u \leq v
\end{aligned}
$$

Since $\mathcal{F}(\theta)=\theta$, so $\mathcal{F}$ satisfies all the conditions of Theorem 3.1, we can conclude that problem (4.1) has a unique positive mild solution.

\section{Conclusion}

In this paper, an equivalent integral equation of a new type for a class of fractional evolution equations is obtained. It is different from those obtained in the existing literature, and according to it, we investigate a class of evolution equations of fractional order with nonlocal conditions on the half-line. Applying the monotone iterative method, without the assumption of lower and upper solutions, we get a new result on the existence and uniqueness of positive mild solutions.

Acknowledgements

This research is supported by the Fundamental Research Funds for the Central Universities (No. 2014QNA52), Natural Science Foundation of Jiangsu Province of China (No. BK20140176), Shandong Provincial Natural Science Foundation (No. ZR2014AP011) and the NNSF (No. 11601508).

Competing interests

The authors declare that they have no competing interests.

Authors' contributions

All of the authors contributed equally in writing this paper.

Author details

${ }^{1}$ School of Mathematics, China University of Mining and Technology, Xuzhou, 221116, China. ${ }^{2}$ School of Business, Central South University, Changsha, 410083, China. ${ }^{3}$ School of Mathematical Sciences, University of Jinan, Jinan, 250022, China. 


\section{Publisher's Note}

Springer Nature remains neutral with regard to jurisdictional claims in published maps and institutional affiliations.

\section{Received: 1 July 2017 Accepted: 8 August 2017 Published online: 18 August 2017}

\section{References}

1. Kilbas, AA, Srivastava, HM, Trujillo, JJ: Theory and Applications of Fractional Differential Equations. Elsevier, Amsterdam (2006)

2. Miller, KS, Ross, B: An Introduction to the Fractional Calculus and Fractional Differential Equations. Wiley, New York (1993)

3. Lakshmikantham, V, Leela, S, Vasundhara Devi, J: Theory of Fractional Dynamic Systems. Cambridge Academic Publishers, Cambridge (2009)

4. Zhou, Y, Ahmad, B, Alsaedi, A: Existence of nonoscillatory solutions for fractional neutral differential equations. Appl. Math. Lett. 72, 70-74 (2017)

5. Zou, Y, He, G: On the uniqueness of solutions for a class of fractional differential equations. Appl. Math. Lett. 74, 68-73 (2017)

6. Zhou, Y: Attractivity for fractional differential equations in Banach space. Appl. Math. Lett. 75, 1-6 (2018). doi:10.1016/j.aml.2017.06.008

7. Raheem, A, Maqbul, MD: Oscillation criteria for impulsive partial fractional differential equations. Comput. Math. Appl. 73, 1781-1788 (2017)

8. Cabada, A, Kisela, T: Existence of positive periodic solutions of some nonlinear fractional differential equations. Commun. Nonlinear Sci. Numer. Simul. 50, 51-67 (2017)

9. Henderson, J, Luca, R: Systems of Riemann-Liouville fractional equations with multi-point boundary conditions. Appl. Math. Comput. 309, 303-323 (2017)

10. Losada, J, Nieto, JJ, Pourhadi, E: On the attractivity of solutions for a class of multi-term fractional functional differential equations. J. Comput. Appl. Math. 312, 2-12 (2017)

11. Dhifli, A, Khamessi, B: Existence and boundary behavior of positive solution for a Sturm-Liouville fractional problem with p-Laplacian. J. Fixed Point Theory Appl. (2017, in press). doi:10.1007/s11784-017-0455-8

12. Mei, DZ, Peng, JG, Gao, JH: General fractional differential equations of order $\alpha \in(1,2)$ and type $\beta \in[0,1]$ in Banach spaces. Semigroup Forum 94, 712-737 (2017)

13. El-Borai, MM: Some probability densities and fundamental solutions of fractional evolution equations. Chaos Solitons Fractals 14, 433-440 (2002)

14. Zhou, Y, Jiao, F: Nonlocal Cauchy problem for fractional evolution equations. Nonlinear Anal., Real World Appl. 11 4465-4475 (2010)

15. Wang, J, Zhou, Y: A class of fractional evolution equations and optimal controls. Nonlinear Anal., Real World Appl. 12, 262-272 (2011)

16. Chen, $\mathrm{P}$, Zhang, PX, Li, Y: Study on fractional non-autonomous evolution equations with delay. Comput. Math. Appl. 73, 794-803 (2017)

17. Chen, $\mathrm{P}, \mathrm{Li}, \mathrm{Y}, \mathrm{Li}, \mathrm{Q}$ : Existence of mild solutions for fractional evolution equations with nonlocal initial conditions. Ann. Pol. Math. 110, 13-24 (2014)

18. Chen, $P, L i, Y, Z$ hang, $X:$ On the initial value problem of fractional stochastic evolution equations in Hilbert spaces. Commun. Pure Appl. Anal. 14, 1817-1840 (2015)

19. Wang, R, Ma, Q: Some new results for multi-valued fractional evolution equations. Appl. Math. Comput. 257, 285-294 (2015)

20. Zhao, J, Wang, R: Mixed monotone iterative technique for fractional impulsive evolution equations. Miskolc Math. Notes 17, 683-696 (2016)

21. Jabeena, T, Lupulescu, V: Existence of mild solutions for a class of non-autonomous evolution equations with nonlocal initial conditions. J. Nonlinear Sci. Appl. 10, 141-153 (2017)

22. Zhou, Y, Shen, $\mathrm{XH}$, Zhang, L: Cauchy problem for fractional evolution equations with Caputo derivative. Eur. Phys. J. Spec. Top. 222, 1749-1765 (2013)

23. Byszewski, L: Existence and uniqueness of a classical solution to a functional-differential abstract nonlocal Cauchy problem. J. Appl. Math. Stoch. Anal. 12, 91-97 (1999)

24. Chen, $P, L i, Y$, Zhang, $X$ : Existence and uniqueness of positive mild solutions for nonlocal evolution equations. Positivity 19, 927-939 (2015)

25. Deimling, K: Nonlinear Functional Analysis. Springer, New York (1985)

26. Guo, D, Lakshmikantham, V: Nonlinear Problems in Abstract Cone. Academic Press, Orlando (1988)

27. Pazy, A: Semigroups of Linear Operators and Applications to Partial Differential Equations. Springer, New York (1983)

28. Engel, K, Nagel, R: One-Parameter Semigroups for Linear Evolution Equations. Springer, New York (1995)

29. Mainardi, F, Paradisi, P, Gorenflo, R: Probability distributions generated by fractional diffusion equations. In: Kertesz, J, Kondor, I (eds.) Econophysics: An Emerging Science. Kluwer Academic, Dordrecht (2000)

30. Hernandez, E, Sakthivel, R, Tanaka, AS: Existence results for impulsive evolution differential equations with state-dependent delay. Electron. J. Differ. Equ. 28, Article ID 1 (2008) 